\title{
ON AN IDENTITY OF ECKFORD COHEN ${ }^{1}$
}

\author{
M. V. SUBBARAO AND D. SURYANARAYANA
}

ABSTRACT. We characterize all multiplicative arithmetical functions $f_{k}(r)$ such that an identity of the form

$$
\sum_{r=1}^{\infty} f_{k}(r) c_{k}(n, r)=q_{k}(n) g(k), \quad g(k) \neq 0,
$$

holds for all $n$, where $q_{k}(n)$ is the characteristic function of the set of $k$-free integers and $c_{k}(n, r)$ is the generalized Ramanujan sum. This characterization yields several arithmetical identities of the above form including an identity of Eckford Cohen, which occurs as a special case of our theorem on taking $f_{k}(r)=\mu(r) / J_{k}(r)$ and $g(k)=\zeta(k)$.

1. Introduction. Throughout the following $k$ denotes a fixed integer $\geqq 2$. Let $Q_{k}$ denote the set of $k$-free integers, that is, the integers whose prime factors are all of multiplicity $<k$. Let $q_{k}(n)=1$ or 0 according as $n \in Q_{k}$ or $n \notin Q_{k}$. Also, let $\zeta(s)=\sum_{n=1}^{\infty} n^{-s}$ for $s>1$.

In 1963, E. Cohen [3, (1)] established the following identity and used it to obtain an elementary estimate for $Q_{k}(x)$, the number of $k$-free integers $\leqq x$ :

$$
\sum_{r=1}^{\infty}\left(\frac{\mu(r)}{J_{k}(r)}\right) c_{k}(n, r)=q_{k}(n) \zeta(k),
$$

where $\mu(r)$ is the Möbius function, $J_{k}(r)$ the Jordan totient function of order $k$, and $c_{k}(n, r)$ is the generalized Ramanujan sum defined by

$$
c_{k}(n, r)=\sum_{a\left(\bmod r^{k}\right) ;\left(a, r^{k}\right)_{k}=1} \exp \left(\frac{2 \pi i a n}{r^{k}}\right),
$$

the summation being over all $a\left(\bmod r^{k}\right)$, whose greatest common $k$ th power divisor with $r^{k}$ is 1 . This generalization of Ramanujan's sum was introduced under a slightly different notation by E. Cohen [2] himself in

Received by the editors June $16,1971$.

AMS 1969 subject classifications. Primary 1043.

Key words and phrases. $k$-free integers, generalized Ramanujan sum, Riemann zeta function.

1 This research is partially supported by NRC Grant A-3103.

(c) American Mathematical Society 1972 
1949, who also established [2, (2.5)] the following arithmetic evaluation of $c_{k}(n, r)$ :

$$
c_{k}(n, r)=\sum_{d\left|r ; d^{k}\right| n} d^{k} \mu(r / d) .
$$

The object of the present paper is to characterize all multiplicative arithmetical functions $f_{k}(r)$ such that an identity of the following type holds for all $n$ :

$$
\sum_{r=1}^{\infty} f_{k}(r) c_{k}(n, r)=q_{k}(n) g(k), \quad g(k) \neq 0 .
$$

It may be noted that (1) is a particular case of (4) with $f_{k}(r)=\mu(r) / J_{k}(r)$ and $g(k)=\zeta(k)$.

The characterization of $f_{i}(r)$ satisfying (4) is given by the following:

THEOREM. Let $f_{k}(r)$ be a multiplicative arithmetic function such that $\sum_{r=1}^{\infty} \hat{f}_{k}(r) c_{k}(n, r)$ is absolutely convergent for all $n$. Then the identity (4) holds if and only if

(i) $f_{k}\left(p^{\alpha}\right)=\left(1+f_{k}(p)\left(p^{k}-1\right)\right) / p^{k}$ for every $\alpha \geqq 2$ and for every prime $p$;

(ii) $g(k) \neq 0$ and is given by $g(k)=\prod_{p}\left\{1-f_{k}(p)\right\}$, where the product is extended over all primes $p$.

REMARK. For the function $\left.f_{k}(r)=\mu(r)\right)_{l} J_{k}(r)$, we note that $f_{k}(p)=$ $-1 /\left(p^{k}-1\right)$ and $f_{k}\left(p^{\alpha}\right)=0$ for every $\alpha \geqq 2$, since $J_{k}(r)=r^{k} \prod_{\nu \mid r}\left(1-1 / p^{k}\right)$; so that the conditions of the theorem are satisfied, thus yielding the identity (1).

2. Proof of the theorem. Since $f_{k}(r)$ and $c_{k}(n, r)$ are multiplicative functions of $r$ and the series $\sum_{r=1}^{\infty} f_{k}(r) c_{k}(n, r)$ is absolutely convergent, it can be expanded into an infinite product of Euler type [4, Theorem 286]. Hence by (3), we have

$$
\begin{aligned}
\sum_{r=1}^{\infty} f_{k}(r) c_{k}(n, r) & =\prod_{p}\left\{\sum_{m=0}^{\infty} f_{k}\left(p^{m}\right) c_{\dot{k}}\left(n, p^{m}\right)\right\} \\
& =\prod_{p}\left\{\sum_{m=0}^{\infty} f_{k}\left(p^{m}\right) \sum_{d\left|p^{m} ; d^{k}\right| n} d^{k} \mu\left(\frac{p^{m}}{d}\right)\right\} .
\end{aligned}
$$

First suppose that $f_{k}\left(p^{\alpha}\right)=\left(1+f_{k}(p)\left(p^{k}-1\right)\right) / p^{k}$, for every $\alpha \geqq 2$ and for every prime $p$, and $g(k) \neq 0$ which is given by $g(k)=\prod_{p}\left\{1-f_{k}(p)\right\}$. Then we prove the identity (4), which is equivalent to proving

$$
\begin{aligned}
\sum_{r=1}^{\infty} f_{k}(r) \mathcal{c}_{k}(n, r) & =\prod_{p}\left\{1-f_{k}(p)\right\}, & & \text { if } n \in Q_{k}, \\
& =0, & & \text { if } n \notin Q_{k} .
\end{aligned}
$$


Now, if $n \in Q_{k}$, then it is clear that 1 is the only $k$ th power divisor of $n$, so that from (5), we get that

$$
\sum_{r=1}^{\infty} f_{k}(r) c_{k}(n, r)=\prod_{p}\left\{\sum_{m=0}^{\infty} f_{k}\left(p^{m}\right) \mu\left(p^{m}\right)\right\}=\prod_{p}\left\{1-f_{k}(p)\right\} .
$$

Let $n \notin Q_{k}$. Then $n$ can be written uniquely in the form $n=n_{1} n_{2}$, where $n_{2}>1,\left(n_{1}, n_{2}\right)=1, n_{1} \in Q_{k}, n_{2} \in L_{k}, L_{k}$ being the set of $k$-full integers, that is, the integers whose prime factors are all of multiplicity $\geqq k$. Let $n_{2}=$ $p_{1}^{\alpha_{1}} p_{2}^{\alpha_{2}} \cdots p_{t}^{\alpha_{t}}$. Since $n_{2} \in L_{k}, \alpha_{i} \geqq k$ for $1 \leqq i \leqq t$. We can write $\alpha_{i}=u_{i} k+v_{i}$, where $u_{i} \geqq 1$ and $0 \leqq v_{i} \leqq k-1$. From (5) we get that

$$
\begin{aligned}
\sum_{r=1}^{\infty} f_{k}(r) c_{k}(n, r)= & \prod_{p}\left\{\sum_{m=0}^{\infty} f_{k}\left(p^{m}\right) \sum_{d\left|p^{m} ; d^{k}\right| n_{1} n_{2}} d^{k} \mu\left(\frac{p^{m}}{d}\right)\right\} \\
= & \prod_{p: p \nmid n_{2}}\left\{\sum_{m=0}^{\infty} f_{k}\left(p^{m}\right) \mu\left(p^{m}\right)\right\} \cdot \prod_{p \mid n_{2}}\left\{\sum_{m=0}^{\infty} f_{k}\left(p^{m}\right) \sum_{d\left|p^{m}: d^{k}\right| n_{2}} d^{k} \mu\left(\frac{p^{m}}{d}\right)\right\} \\
= & \prod_{p: p \nmid n_{2}}\left\{1-f_{k}(p)\right\} \cdot \prod_{i=1}^{t}\left\{\sum_{m=0}^{\infty} f_{k}\left(p^{m}\right) \sum_{d\left|p_{i}^{m} ; d^{k}\right| p_{i} \alpha_{i}} d^{k} \mu\left(\frac{p_{i}^{m}}{d}\right)\right\} \\
= & \prod_{p: p \nmid n_{2}}\left\{1-f_{k}(p)\right\} \\
& \cdot \prod_{i=1}^{t}\left\{1+\sum_{a=1}^{u_{i}} f_{k}\left(p_{i}^{n}\right)\left[p_{i}^{a k}-p_{i}^{(a-1) k}\right]-f_{k}\left(p_{i}^{u_{i}+1}\right) p_{i}^{u_{i k} k}\right\} .
\end{aligned}
$$

Since $f_{k}\left(p^{\alpha}\right)=\left(1+f_{k}(p)\left(p^{k}-1\right)\right) / p^{k}$ for every $\alpha \geqq 2$ and for every prime $p$, we have

$$
f_{k}\left(p_{i}^{2}\right)=f_{k}\left(p_{i}^{3}\right)=\cdots=f_{k}\left(p_{i}^{u_{i}+1}\right)=\left(1+f_{k}\left(p_{i}\right)\left(p_{i}^{k}-1\right)\right) / p_{i}^{k}
$$

for $1 \leqq i \leqq t$. Hence each term in the second product is zero, so that

$$
\sum_{r=1}^{\infty} f_{k}(r) c_{k}(n, r)=0 \text { for all } n \notin Q_{k} .
$$

Thus (6) is proved, so that the first part of the theorem follows.

Conversely, suppose that (4) holds for all $n$ with $g(k) \neq 0$. Taking $n=1$, we see from (3) that $c_{k}(1, r)=\mu(r)$, so that from (4) and (5), we get that

$$
g(k)=\sum_{r=1}^{\infty} f_{k}(r) \mu(r)=\prod_{p}\left\{1-f_{k}(p)\right\},
$$

so that condition (ii) of the theorem is satisfied. Since $g(k) \neq 0$, we have $f_{k}(p) \neq 1$ for every prime $p$.

Let $q$ be any arbitrary prime. To prove condition (i) of the theorem, 
it is enough if we prove that

$$
f_{k}\left(q^{\alpha}\right)=\left(1+f_{k}(q)\left(q^{k}-1\right)\right) / q^{k} \text { for every } \alpha \geqq 2 \text {. }
$$

We prove (8) by complete induction on $\alpha$.

Now, taking $n=q^{k}$ in (4), we get from (7) (in this case $n_{1}=1, n_{2}=q^{k}$, $i=1$ and $u_{1}=1$ ) that

$$
\begin{aligned}
0 & =\prod_{p ; p \neq q}\left\{1-f_{k}(p)\right\} \cdot\left\{1+f_{k}(q)\left(q^{k}-1\right)-f_{k}\left(q^{2}\right) q^{k}\right\} \\
& =\left[g(k) /\left(1-f_{k}(q)\right)\right] \cdot\left\{1+f_{k}(q)\left(q^{k}-1\right)-f_{k}\left(q^{2}\right) q^{k}\right\} .
\end{aligned}
$$

Since $g(k) \neq 0$, we get that

$$
f_{k}\left(q^{2}\right)=\left(1+f_{i}(q)\left(q^{k}-1\right)\right) / q^{k},
$$

that is, (8) is true for $\alpha=2$.

Suppose that (8) is true for $\alpha=2,3, \cdots, \beta$, where $\beta \geqq 2$. We shall prove (8) for $\alpha=\beta+1$. Taking $n=q^{\beta k}$ in (4), we get from (7) (in this case $n_{1}=1$, $n_{2}=q^{\beta k}, i=1$ and $u_{1}=\beta$ ) that

$$
0=\prod_{p: p \neq q}\left\{1-f_{k}(p)\right\} \cdot\left\{1+\sum_{a=1}^{\beta} f_{\hat{k}}\left(q^{a}\right)\left[q^{a k}-q^{(a-1) k}\right]-f_{k}\left(q^{\beta+1}\right) q^{\beta k}\right\},
$$

so that

$$
\begin{aligned}
q^{\beta k} f_{k}\left(q^{\beta+1}\right) & =1+f_{k}(q)\left(q^{k}-1\right)+\sum_{a=2}^{\beta} f_{k}\left(q^{a}\right)\left[q^{a k}-q^{(a-1) k}\right] \\
& =1+f_{k}(q)\left(q^{k}-1\right)+f_{k}\left(q^{2}\right)\left[q^{\beta k}-q^{k}\right] \\
& =1+f_{k}(q)\left(q^{k}-1\right)+\left[\frac{1+f_{k}(q)\left(q^{k}-1\right)}{q^{k}}\right]\left(q^{\beta k}-q^{k}\right) \\
& =q^{(\beta-1) k}\left[1+f_{k}(q)\left(q^{k}-1\right)\right],
\end{aligned}
$$

that is, $f_{k}\left(q^{\beta+1}\right)=\left(1+f_{k}(q)\left(q^{k}-1\right)\right) / q^{k}$. Hence (8) follows for every $\alpha \geqq 2$. Thus the proof of the theorem is complete.

3. Some special cases. Taking

$$
f_{k}(p)=\frac{A}{p^{k}-B} \text { and } f_{k}\left(p^{\alpha}\right)=\frac{(A+1) p^{k}-(A+B)}{p^{k}\left(p^{k}-B\right)}
$$

for every $\alpha \geqq 2$ and for every prime $p$, where $A$ and $B$ are constants which have the values 0,1 or -1 , we see that the conditions of the theorem are satisfied with $g(k)=\prod_{p}\left\{1-A /\left(p^{k}-B\right)\right\}$. Hence we have the following identities corresponding to the values of $A$ and $B$ given by $(1,0),(1,-1)$, 
$(1,1),(-1,0),(-1,-1),(-1,1)$ :

$$
\begin{aligned}
& \sum_{r=1}^{\infty} f_{k}(r) c_{k}(n, r)=\frac{q_{k}(n)}{\zeta(k)}, \\
& \sum_{r=1}^{\infty} f_{k}(r) c_{k}(n, r)=q_{k}(n) \frac{\zeta(2 k)}{\zeta(k)}, \\
& \sum_{r=1}^{\infty} f_{k}(r) c_{k}(n, r)=q_{k}(n) \zeta(k) \prod_{p}\left(1-\frac{2}{p^{k}}\right), \\
& \sum_{r=1}^{\infty} f_{k}(r) c_{k}(n, r)=q_{k}(n) \frac{\zeta(k)}{\zeta(2 k)}, \\
& \sum_{r=1}^{\infty} f_{k}(r) c_{k}(n, r)=q_{k}(n) \frac{\zeta(2 k)}{\zeta(k)} \prod_{p}\left(1+\frac{2}{p^{k}}\right), \\
& \sum_{r=1}^{\infty} f_{k}(r) c_{k}(n, r)=q_{k}(n) \zeta(k) .
\end{aligned}
$$

The infinite products in the right sides of (11) and (13) can be expressed as infinite products involving the Riemann zeta function using the following result.

$$
\prod_{p}\left(1-\frac{\alpha}{p^{s}}\right)^{-1}=\prod_{m=1}^{\infty}(\zeta(m s))^{a(m)},
$$

for every $\alpha$ and $s>1$, where $a(m)=m^{-1} \sum_{d \mid m} \alpha^{d} \mu(m / d)$.

For a proof of this result, we refer to [1].

\section{REFERENCES}

1. L. Carlitz and M. V. Subbarao, On a class of multipiicative functions, Duke Math. J. (to appear).

2. E. Cohen, An extension of Ramanujan's sum, Duke Math. J. 16 (1949), 85-90. MR 10, 354.

3. - An elementary estimate for the $k$-free integers, Bull. Amer. Math. Soc. 69 (1963), 762-765. MR 27 \#3591.

4. G. H. Hardy and E. M. Wright, An introduction to the theory of numbers, 4th ed., Oxford Univ. Press, London, 1960.

Department of Mathematics, University of Alberta, Edmonton, Canada

Current address (D. Suryanarayana): Department of Mathematics, Andhra University, Waltair, India 